\title{
An Arithmetic and Combinatorial Approach to Three-Dimensional Discrete Lines
}

\author{
Valérie Berthé ${ }^{1}$ and Sébastien Labbéé, \\ 1 Laboratoire d'Informatique Algorithmique : Fondements et Applications, \\ Université Paris Diderot, Paris 7 - Case 7014 \\ F-75205 Paris Cedex 13, France \\ berthe@liafa.jussieu.fr \\ 2 Laboratoire de Combinatoire et d'Informatique Mathématique, \\ Université du Québec à Montréal, \\ C.P. 8888 Succursale "Centre-Ville", Montréal (QC), Canada H3C 3P8 \\ labbe.sebastien@courrier.uqam.ca
}

\begin{abstract}
The aim of this paper is to discuss from an arithmetic and combinatorial viewpoint a simple algorithmic method of generation of discrete segments in the three-dimensional space. We consider discrete segments that connect the origin to a given point $\left(u_{1}, u_{2}, u_{3}\right)$ with coprime nonnegative integer coordinates. This generation method is based on generalized three-dimensional Euclid's algorithms acting on the triple $\left(u_{1}, u_{2}, u_{3}\right)$. We associate with the steps of the algorithm substitutions, that is, rules that replace letters by words, which allow us to generate the Freeman coding of a discrete segment. We introduce a dual viewpoint on these objects in order to measure the quality of approximation of these discrete segments with respect to the corresponding Euclidean segment. This viewpoint allows us to relate our discrete segments to finite patches that generate arithmetic discrete planes in a periodic way.
\end{abstract}

Keywords: Discrete Segments, Discrete Lines, Christoffel words, multidimensional Euclid's algorithms, multi-dimensional continued fractions, substitutions.

\section{Introduction}

Discrete lines and segments in the plane are quite well understood and their study has already arised a vast literature on the subject (see e.g. the references in 14]). The Freeman codings of arithmetic standard discrete lines correspond to the also well-studied family of Sturmian words. For more details, see e.g. [15]. Among the factors of Sturmian words, Christoffel words play a particular role and correspond to Freeman codings of segments. The deep and fruitful connections between Sturmian words and continued fractions, on the one hand, and between Christoffel words and Euclid's algorithm, on the other hand, allows a thorough description of most of their properties (see Figure 1 for an illustration).

\footnotetext{
${ }^{\star}$ With support of the NSERC. The authors also would like to thank P. Arnoux and X.
} Provençal for many fruitful discussions, and the referees for their valuable remarks. 


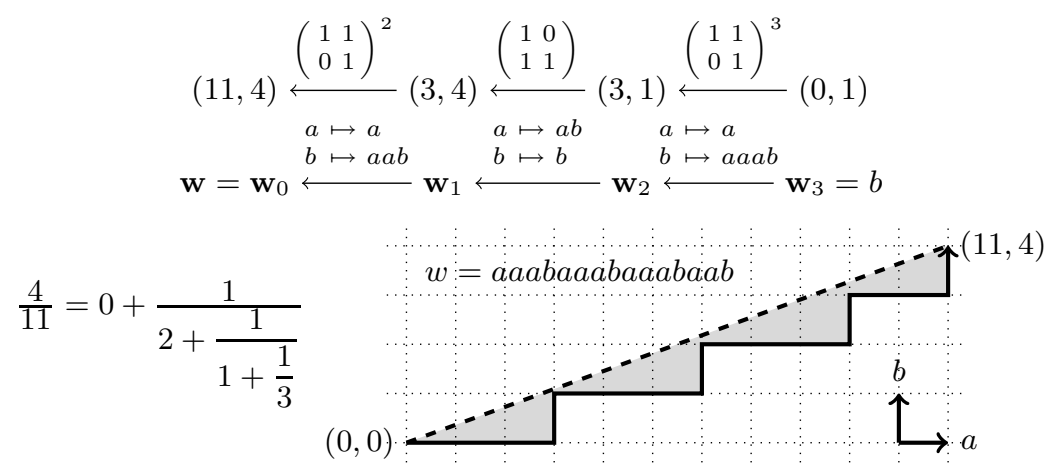

Fig. 1. It is well known that the reduction of a two-dimensional integer vector by using Euclid's algorithm allows one to construct the discrete segment (also called Christoffel word) which is such that no integer point is in the interior of the gray region. Its Freeman coding $\mathbf{w}=a a a b a a a b a a a b a a b$ can be obtained by applying on the letter $b$ the substitutions associated with the steps of Euclid's algorithm performed on $(11,4)$.

There exist various strategies for defining and generating discrete lines in the three-dimensional space. With no claim for being exhaustive, let us quote e.g. [1]12 18 8 89 . The approach we follow here is motivated by the study of Sturmian words. Several generalizations of Sturmian words over a three-letter alphabet have been considered. For instance, infinite words coding trajectories in a cubic billiard have been investigated in [3]. One of their drawbacks is that they produce infinite words having a quadratic number of factors of a given length, which seems to indicate that there is no suitable continued fraction algorithm allowing one to describe them. This prevents in particular multiscale analysis. Another direction of generalization of Sturmian words consists in working with balanced words over a three-letter alphabet. However balanced words over a higher-alphabet do not seem to be good candidates for describing discrete segments in the space, as shown in [13. The family of Arnoux-Rauzy words [4 provides a third fruitful way of generalizing Sturmian words. They have a linear number of factors of a given length $(2 n+1$ factors of length $n)$, and can be described in terms of a multi-dimensional continued fraction algorithm. Nevertheless this algorithm is not defined everywhere, and thus, they cannot be used to approximate all the slopes in the space. For more details, see the discussion and the references in [5.

Our strategy here works in the reverse direction: we start from Euclid's algorithms that are defined everywhere and we associate with them families of words. More precisely, we want to construct discrete segments that connect the origin to a given point $\left(u_{1}, u_{2}, u_{3}\right)$ with coprime nonnegative integer coordinates. We apply a three-dimensional Euclid's algorithm to the triple $\left(u_{1}, u_{2}, u_{3}\right)$ (see Section 2.1). In Section 2.2 we associate with the steps of the algorithm substitutions, that is, rules that replace letters by words, which allow us to generate the Freeman coding of the discrete segment. 
We thus obtain in Section 3 a simple algorithmic way for producing discrete segments and lines by means of substitutions and generalized Euclid's algorithms which can allow a multiscale approach for their study. Our description is both analytic and arithmetic. Note that by discrete segment, we mean here broken lines constructed by concatenating unit steps oriented along the three coordinate axes. Based on the quality of approximation of the underlying multi-dimensional continued fraction algorithms that are used (see Remark 2 below), we expect these segments to be good candidates for discretizations of Euclidean segments and lines, which is supported by the experimental studies we conducted.

Section 4 aims at getting a theoretical and dynamical understanding of the way these segments approximate Euclidean segments by introducing a discrete plane that is transverse to the original direction $\left(u_{1}, u_{2}, u_{3}\right)$. The present paper relies on a formalism that has been previously introduced in a different context in [2. The new notions that are introduced here correspond mainly to Definition 3 and to the choice of the normal vector $\boldsymbol{v}$ of the transverse plane in Equation (1).

\section{Preliminaries}

Let $\boldsymbol{u}=\left(u_{1}, u_{2}, u_{3}\right)$ be a vector with coprime entries in $\mathbb{N}^{3}$. We want to introduce a discrete line approximating the vectorial line directed by the vector $\boldsymbol{u}$ in $\mathbb{R}^{3}$.

\subsection{Generalized Euclid's Algorithm}

In the one-dimensional case, most of the existing continued fraction algorithms strongly rely on Euclid's algorithm: starting from two nonnegative numbers $a$ and $b$, one subtracts the smallest one to the largest one. If one performs only one subtraction at each step, one obtains the so-called additive version of Euclid's algorithm. If one performs in one step as much subtractions as possible (i.e., if $0 \leq b \leq a, a$ is replaced by $a-[a / b] b)$, one gets a multiplicative algorithm. In the multi-dimensional case, there is no such canonical algorithm, and several different definitions have been proposed (see [17] for a summary). Indeed starting from more than two numbers, it is not clear to decide which operation is to be performed on these numbers, hence the diversity of existing generalizations of Euclid's algorithm (see Section 2.3).

We will thus use the following framework for defining versions of threedimensional generalizations of Euclid's algorithms. Let $\mathcal{M}_{E}$ be the set of $3 \times 3$ matrices $M=\left[m_{i j}\right]_{1 \leq i, j \leq 3}$ with entries in $\{0,1\}$ having only 1 's on the diagonal and exactly one nonzero entry $m_{i j}$ with $i \neq j$. Let $\mathcal{M}_{P}$ be the set of $3 \times 3$ matrices that are permutation matrices, that is, they have entries in $\{0,1\}$, and only one nonzero coefficient on each line and on each column. Matrices of $\mathcal{M}_{E}$ and of $\mathcal{M}_{P}$ are called elementary matrices. We set $\mathcal{M}$ to be the set of finite products of matrices of $\mathcal{M}_{E} \cup \mathcal{M}_{P}$.

\section{Definition 1 (Three-dimensional Euclid's algorithm). Let}

$$
X \subset\left\{\left(u_{1}, u_{2}, u_{3}\right) \mid \forall i, u_{i} \in \mathbb{N}, \text { and } \operatorname{gcd}\left(u_{1}, u_{2}, u_{3}\right)=1\right\}
$$

and let $X_{f} \subset X$. Elements of $X_{f}$ are called terminal. 
A three-dimensional Euclid's algorithm is a map $T: X \rightarrow X$ such that $T(x)=$ $x$ for all $x \in X_{f}$, for any $\boldsymbol{u} \in X$ there is $M \in \mathcal{M}$ satisfying $\boldsymbol{u}=M T(\boldsymbol{u})$, and for every $\boldsymbol{u} \in X$ there exists $N$ such that $T^{N}(\boldsymbol{u}) \in X_{f}$.

\subsection{Euclid's Substitutions}

Let us consider a finite set of letters $\mathcal{A}$ called alphabet. A (finite) word is an element of the free monoid $\mathcal{A}^{*}$ generated by $\mathcal{A}$. A substitution $\sigma$ over the alphabet $\mathcal{A}$ is an endomorphism of the free monoid $\mathcal{A}^{*}$. It is completely defined by its image on the letters of the alphabet. For $i \in\{1,2,3\}$ and for $w \in\{1,2,3\}^{*}$, let $|w|_{i}$ stand for the number of occurrences of the letter $i$ in the word $w$. The map

$$
\boldsymbol{l}:\{1,2,3\}^{*} \rightarrow \mathbb{N}^{n}, w{ }^{t}\left(|w|_{1},|w|_{2},|w|_{3}\right)
$$

is called the abelianization map. Notice that in the literature, this map is also referred to as the Parikh mapping. Let $\sigma$ be a substitution on $\{1,2,3\}^{*}$. Its incidence matrix or abelianized matrix $M_{\sigma}=\left(m_{i, j}\right)_{1 \leq i, j \leq 3}$ is defined as the square matrix with entries $m_{i, j}=|\sigma(j)|_{i}$ for all $i, j$. We say that $\sigma$ is unimodular if $\operatorname{det}\left(M_{\sigma}\right)= \pm 1$.

Definition 2 (Three-dimensional Euclid's substitutions). Let $T$ be a threedimensional Euclid's algorithm. With each matrix $M \in \mathcal{M}$ produced by the algorithm, we associate a substitution whose incidence matrix is given by $M$.

Remark 1. Given a matrix produced by a Euclid's algorithm, there exist several substitutions having this matrix as incidence matrix. The substitutions generating words that are Freeman codings of discrete segments are known to be Sturmian. Given an incidence matrix $\left[\begin{array}{ll}a & b \\ c & d\end{array}\right]$ of a Sturmian substitution, only $a+b+c+d-1$ substitutions having this matrix as incidence matrix are Sturmian (i.e., preserve discrete segments). For more details, see [15] and the references therein. Hence, the choice of a substitution associated with an incidence matrix can play an important role. This is why we try to privilege as much as possible here additive steps. We thus usually recover elementary matrices or simple products of them, which reduces the choices for the associated substitution.

Example 1. With the elementary matrix $M=\left[\begin{array}{lll}1 & 0 & 0 \\ 1 & 1 & 0 \\ 0 & 0 & 1\end{array}\right]$ are associated $\sigma: 1 \mapsto 12,2 \mapsto 2,3 \mapsto 3$ and $\tilde{\sigma}: 1 \mapsto 21,2 \mapsto 2,3 \mapsto 3$

\subsection{A Zoo of Algorithms}

We recall here the most classical generalizations of Euclid's algorithms which have lead to well-studied multi-dimensional continued fraction algorithms such as those discussed in [17]: 
- Jacobi-Perron: let $0 \leq u_{1}, u_{2} \leq u_{3}$

$$
\left(u_{1}, u_{2}, u_{3}\right) \mapsto\left(u_{2}-\left[\frac{u_{2}}{u_{1}}\right] u_{1}, u_{3}-\left[\frac{u_{3}}{u_{1}}\right] u_{1}, u_{1}\right),
$$

- Brun: we subtract the second largest entry to the largest one; for instance, if $0 \leq u_{1} \leq u_{2} \leq u_{3}$,

$$
\left(u_{1}, u_{2}, u_{3}\right) \mapsto\left(u_{1}, u_{2}, u_{3}-u_{2}\right) ;
$$

- Poincaré: we subtract the second largest entry to the largest one, and the smallest entry to the second largest one; for instance, if $0 \leq u_{1} \leq u_{2} \leq u_{3}$

$$
\left(u_{1}, u_{2}, u_{3}\right) \mapsto\left(u_{1}, u_{2}-u_{1}, u_{3}-u_{2}\right),
$$

- Selmer: we subtract the smallest positive entry to the largest one; for instance, if $0<u_{1} \leq u_{2} \leq u_{3}$

$$
\left(u_{1}, u_{2}, u_{3}\right) \mapsto\left(u_{1}, u_{2}, u_{3}-u_{1}\right),
$$

- Fully subtractive: we subtract the smallest positive entry to all the largest ones; for instance, if $0<u_{1} \leq u_{2} \leq u_{3}$

$$
\left(u_{1}, u_{2}, u_{3}\right) \mapsto\left(u_{1}, u_{2}-u_{1}, u_{3}-u_{1}\right) .
$$

We have recalled here Jacobi-Perron algorithm in its multiplicative form for the sake of clarity, but an additive version of this algorithm can be given. Furthermore, one checks that we can chose as terminal set for all these algorithms the set

$$
X_{f}=\{(1,0,0),(0,1,0),(0,0,1)\} \subset X,
$$

by possibly applying the two-dimensional Euclid's algorithm once the first coordinate has reached the value 0 in the Jacobi-Perron case.

Remark 2. The choice of these algorithms is motivated by the quality of approximation they provide. Indeed, Jacobi-Perron and Brun algorithm are known to provide almost everywhere exponential convergence (see [10]).

Example 2. Let $\boldsymbol{u}=(2,2,3)$. By using Brun algorithm, one has $\boldsymbol{u}_{0}=(2,2,3)$, $\boldsymbol{u}_{1}=(2,2,1), \boldsymbol{u}_{2}=(0,2,1), \boldsymbol{u}_{3}=(0,1,1), \boldsymbol{u}_{4}=(0,0,1)$. By using Poincaré algorithm, one obtains $\boldsymbol{u}_{0}=(2,2,3), \boldsymbol{u}_{1}=(2,0,1), \boldsymbol{u}_{2}=(1,0,1), \boldsymbol{u}_{3}=(1,0,0)$.

\section{A Generation Method for Discrete Segments}

Let us apply to $\boldsymbol{u}$ a finite sequence of steps under the action of one of the threedimensional Euclid's algorithm $T$ given in Section 2.3 with $X_{f}$ defined as above together with a choice of Euclid's substitutions associated with the produced matrices. One has $\boldsymbol{u}=M_{1} \cdots M_{N} \boldsymbol{u}_{N}$, where the vector $\boldsymbol{u}_{N} \in X_{f}$ has only two coordinates equal to 0 , and one coordinate equal to 1 . Let $\mathbf{w}_{N} \in\{1,2,3\}$ be the unique word (of length one) such that $\boldsymbol{l}\left(\mathbf{w}_{N}\right)=\boldsymbol{u}_{N}$. The associated Euclid's substitutions are denoted by $\sigma_{n}$, for $1 \leq n \leq N$ (see the diagram below). 


$$
\begin{gathered}
\boldsymbol{u}=\boldsymbol{u}_{0} \stackrel{M_{1}^{-1}}{\longrightarrow} \boldsymbol{u}_{1} \stackrel{M_{2}^{-1}}{\longrightarrow} \boldsymbol{u}_{2} \stackrel{M_{3}^{-1}}{\longrightarrow} \cdots \stackrel{M_{N}^{-1}}{\longrightarrow} \boldsymbol{u}_{N} \in X_{f} \\
\mathbf{w}=\mathbf{w}_{0} \stackrel{\sigma_{1}}{\longleftarrow} \mathbf{w}_{1} \stackrel{\sigma_{2}}{\longleftarrow} \mathbf{w}_{2} \stackrel{\sigma_{3}}{\longleftarrow} \cdots \stackrel{\sigma_{N}}{\longleftarrow} \mathbf{w}_{N} \in\{1,2,3\}
\end{gathered}
$$

Definition 3 (Discrete segment). The discrete segment associated with the vector $\boldsymbol{u}$ and with the three-dimensional Euclid's algorithm $T$ is defined as the broken line with integer vertices that starts from the origin, whose Freeman coding is given by the coding word

$$
\mathbf{w}:=\sigma_{1} \cdots \sigma_{N}\left(\mathbf{w}_{N}\right) .
$$

In other words, the vertices of this broken line are given by the abelianized by $\boldsymbol{l}$ of the prefixes of the word $\mathbf{w}$.

Example 3. If $\boldsymbol{u}=(2,2,3)$ and by using Poincaré's algorithm, one has $\mathbf{w}_{N}=$ $\mathbf{w}_{3}=1$ and $\mathbf{w}=\mathbf{w}_{0}=1231233$.

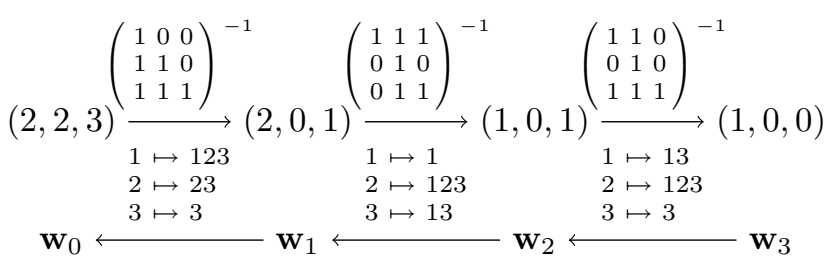

The discrete segment is depicted below. Its Euclidean distance to the Euclidean segment is 1.3720 .

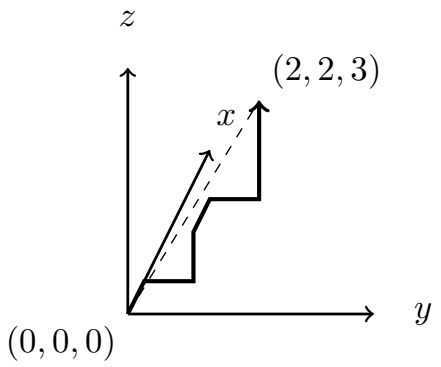

\section{A Dual Viewpoint}

In this section, we introduce the following notation: one sets $M_{i . . j}:=M_{i} \cdots M_{j}$ and $\sigma_{i . . j}:=\sigma_{i} \cdots \sigma_{j}$ for $1 \leq i, j \leq N$. Hence, the incidence matrix of the substitution $\sigma_{i . . j}$ is $M_{i . . j}$.

In order to study the quality of approximation of the vector line directed by $\mathbf{u}$ provided by the discrete segment $\mathbf{w}$, we introduce a transverse plane that does 
not contain vector $\boldsymbol{u}$. Such a plane can be described by its normal vector $\boldsymbol{v}$ that we chose with positive entries and not collinear with $\boldsymbol{u}$. The vector having all entries equal to 1 is denoted by $\mathbf{1}$. We chose for $\boldsymbol{v}$

$$
\boldsymbol{v}:={ }^{t} M_{1 . . N} \cdot \mathbf{1}={ }^{t} M_{N} \cdot{ }^{t} M_{1} \cdot \mathbf{1}
$$

We furthermore write $\mathbf{w}=z_{1} \cdots z_{k} \cdots z_{|\mathbf{w}|}$ where $z_{k} \in\{1,2,3\}$ are letters. The vertices of the discrete segment are thus of the form $p_{k}=\boldsymbol{l}\left(z_{1} \cdots z_{k}\right)$, for $1 \leq$ $k \leq|\mathbf{w}|$. The choice of vector $\boldsymbol{v}$ is motivated by the following relation that we will use below

$$
\begin{aligned}
\left\langle p_{k}, \mathbf{1}\right\rangle & =\left\langle\left(M_{1 . . N}\right)^{-1} \cdot p_{k},{ }^{t} M_{1 . . N} \cdot \mathbf{1}\right\rangle \\
& =\left\langle\left(M_{1 . . N}\right)^{-1} \cdot p_{k}, \boldsymbol{v}\right\rangle .
\end{aligned}
$$

The aim of this section is to relate vertices of the discrete segment to faces of a finite pattern of the discrete plane with normal vector $\boldsymbol{v}$ via the mapping $\left(M_{1 . . N}\right)^{-1}$, and to interpret the coding word $\mathbf{w}$ in terms of a coding of the orbit of a point under a dynamical system acting on this discrete plane with normal vector $\boldsymbol{v}$. For that purpose, we introduce in Section 4.2 a dual notion of substitution acting on faces of discrete planes.

\subsection{Discrete Planes}

Let $\boldsymbol{n}$ be a nonzero vector in $\mathbb{N}^{3}$. According to [16], we recall that the arithmetic standard plane $\mathfrak{P}_{\boldsymbol{n}}$ of normal vector $\boldsymbol{n}=\left(n_{1}, n_{2}, n_{3}\right)$ is defined as

$$
\mathfrak{P}_{\boldsymbol{n}}=\left\{\boldsymbol{x} \in \mathbb{Z}^{3} \mid 0<\langle\boldsymbol{x}, \boldsymbol{n}\rangle \leq\|\boldsymbol{n}\|_{1}=n_{1}+n_{2}+n_{3}\right\} .
$$

For $\boldsymbol{x} \in \mathbb{Z}^{3}$ and $i \in\{1,2,3\}$, let $\left(\boldsymbol{x}, i^{*}\right)$ stand for the pointed face defined as the translation by $\boldsymbol{x}$ of the surfel generated by $\left\{\boldsymbol{e}_{1}, \boldsymbol{e}_{2}, \boldsymbol{e}_{3}\right\} \backslash\left\{\boldsymbol{e}_{i}\right\}$ (see Figure 2). We say that $\boldsymbol{x}$ is the vertex and $i$ is the type of the pointed face $\left(\boldsymbol{x}, i^{*}\right)$.

$$
\begin{aligned}
& \left(\boldsymbol{x}, 1^{*}\right):=\boldsymbol{x}+\left\{\lambda \boldsymbol{e}_{2}+\mu \boldsymbol{e}_{3},(\lambda, \mu) \in[0,1]^{2}\right\} \\
& \left(\boldsymbol{x}, 2^{*}\right):=\boldsymbol{x}+\left\{\lambda \boldsymbol{e}_{1}+\mu \boldsymbol{e}_{3},(\lambda, \mu) \in[0,1]^{2}\right\} \\
& \left(\boldsymbol{x}, 3^{*}\right):=\boldsymbol{x}+\left\{\lambda \boldsymbol{e}_{1}+\mu \boldsymbol{e}_{2},(\lambda, \mu) \in[0,1]^{2}\right\}
\end{aligned}
$$

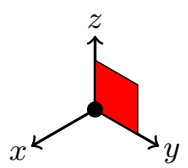

$\left(\mathbf{0}, 1^{*}\right)$

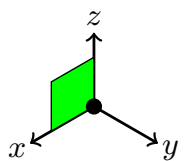

$\left(\mathbf{0}, 2^{*}\right)$

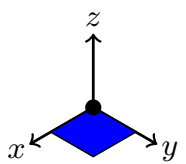

$\left(\mathbf{0}, 3^{*}\right)$
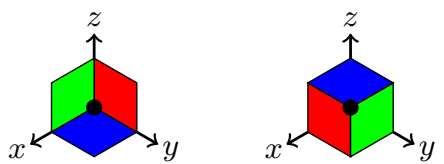

Fig. 2. Left: Geometric interpretation of faces. Right: Lower unit cube and upper unit cube. In this figure and the following, the vertex $(0,0,0)$ or $(1,1,1)$ is identified by a black dot. 
We will use the following notation for translates of faces: if $\left(\boldsymbol{x}, i^{*}\right)$ is a face and $\boldsymbol{y}$ is a vector, then $\left(\boldsymbol{x}, i^{*}\right)+\boldsymbol{y}:=\left(\boldsymbol{x}+\boldsymbol{y}, i^{*}\right)$ which extends in a natural way to union of faces. The lower unit cube refers to the set $\left\{\left(\mathbf{0}, 1^{*}\right),\left(\mathbf{0}, 2^{*}\right),\left(\mathbf{0}, 3^{*}\right)\right\}$, whereas the upper unit cube refers to $\left\{\left(\boldsymbol{e}_{1}, 1^{*}\right),\left(\boldsymbol{e}_{2}, 2^{*}\right),\left(\boldsymbol{e}_{3}, 3^{*}\right)\right\}$ (see Figure 2).

Let $\mathcal{P}_{\boldsymbol{n}}$ be the set of pointed faces satisfying

$$
\mathcal{P}_{\boldsymbol{n}}=\left\{\left(\boldsymbol{x}, i^{*}\right) \mid 0 \leq\langle\boldsymbol{x}, \boldsymbol{n}\rangle<n_{i}\right\} .
$$

One checks that the points of $\mathfrak{P}_{\boldsymbol{n}}$ are the vertices (i.e., the corners) of the faces of $\mathcal{P}_{\boldsymbol{n}}$. By abuse of terminology, by arithmetic discrete plane with normal vector $\boldsymbol{n}$, we mean in all that follows this union of pointed faces $\mathcal{P}_{\boldsymbol{n}}$. Note that in particular, if $\boldsymbol{n}$ has positive entries, the lower unit cube is included in $\mathcal{P}_{\boldsymbol{n}}$.

Furthermore, for any vertex $p_{k}=\boldsymbol{l}\left(z_{1} \cdots z_{k}\right)$ of the discrete segment, one has

$$
0 \leq\left\langle p_{k}, \mathbf{1}\right\rangle \leq\langle\boldsymbol{u}, \mathbf{1}\rangle=\left\langle M_{1 . . N} \cdot \boldsymbol{u}_{N}, \mathbf{1}\right\rangle=\left\langle\boldsymbol{u}_{N},{ }^{t} M_{1 . . N} \cdot \mathbf{1}\right\rangle=\left\langle\boldsymbol{u}_{N}, \boldsymbol{v}\right\rangle=v_{\mathbf{w}_{N}} .
$$

Hence by (2) and (3) the vertices $p_{k}$ of the discrete segment are mapped by $\left(M_{1 . . N}\right)^{-1}$ onto vertices of faces of type $\mathbf{w}_{N}$ of the discrete plane $\mathcal{P}_{\boldsymbol{v}}$. The aim of the next section is to investigate this relation.

\subsection{Generalized Substitutions}

When $\sigma$ is a unimodular substitution, it is possible to associate with it a notion of substitution acting on faces of cubes, following the formalism of [2]:

$$
E_{1}^{*}(\sigma)\left(\boldsymbol{x}, i^{*}\right):=\sum_{j \in\{1,2,3\}} \sum_{p, s \text { such that } \sigma(j)=p i s}\left(M_{\sigma}^{-1}(\boldsymbol{x}+\boldsymbol{l}(s)), j^{*}\right) .
$$

The action of $E_{1}^{*}(\sigma)$ extends in a natural way to unions of faces. A mapping of the form $E_{1}^{*}(\sigma)$ is called a generalized substitution. It is obtained as the dual map of some map $E_{1}(\sigma)$ that can be seen as a geometric realization of $\sigma$. In the notation $E_{1}^{*}(\sigma)$, the subscript of $E_{1}^{*}(\sigma)$ stands for the codimension of the faces, while the superscript of $E_{1}^{*}(\sigma)$ refers to duality. Note that the incidence matrix of $E_{1}^{*}(\sigma)$ is the transpose of the incidence matrix of $\sigma$.

$$
1 \mapsto 123 \quad 1 \mapsto 1 \quad 1 \mapsto 13
$$

Example 4. Let $\sigma_{1}: 2 \mapsto 23, \sigma_{2}: 2 \mapsto 123, \sigma_{3}: 2 \mapsto 123$ be the substitutions

$$
3 \mapsto 3 \quad 3 \mapsto 13 \quad 3 \mapsto 3
$$

obtained from the reduction of the vector $(2,2,3)$ by using Poincaré algorithm. If $\mathcal{C}=$ is the lower unit cube, then one gets
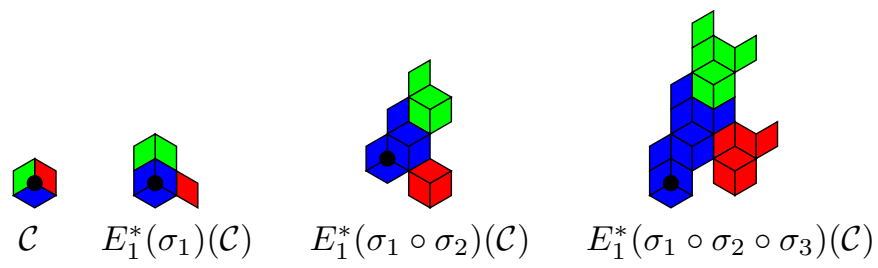
One key property of generalized substitutions is that they preserve discrete planes, as proved in [2]. Indeed, one has the following result for any unimodular substitution $\sigma$ and any vector $\boldsymbol{n}$ with nonnegative entries:

$$
E_{1}^{*}(\sigma)\left(\mathcal{P}_{\boldsymbol{n}}\right)=\mathcal{P}_{t^{t}}{ }_{\sigma} \boldsymbol{n} .
$$

\subsection{Dual Pattern}

We can now apply the notions previously introduced in order to define a pattern of the discrete plane $\mathcal{P}_{\boldsymbol{v}}$ (see (11)) that can be associated with the coding word $\mathbf{w}$.

Definition 4 (Dual pattern). For $i=\{1,2,3\}$, let

and

$$
\mathcal{W}_{i}:=E_{1}^{*}\left(\sigma_{N}\right) \circ \cdots \circ E_{1}^{*}\left(\sigma_{1}\right)\left(\mathbf{0}, i^{*}\right)
$$

$$
\mathcal{W}_{i}^{\prime}:=E_{1}^{*}\left(\sigma_{N}\right) \circ \cdots \circ E_{1}^{*}\left(\sigma_{1}\right)\left(\boldsymbol{e}_{i}, i^{*}\right) .
$$

One sets furthermore $\mathcal{W}=\mathcal{W}_{1} \cup \mathcal{W}_{2} \cup \mathcal{W}_{3}$ and $\mathcal{W}^{\prime}=\mathcal{W}_{1}^{\prime} \cup \mathcal{W}_{2}^{\prime} \cup \mathcal{W}_{3}^{\prime}$.

According to 2], the three patterns $\mathcal{W}_{i}$ (resp. $\mathcal{W}_{i}^{\prime}$ ) for $i \in\{1,2,3\}$ have disjoint interiors. Furthermore $\mathcal{W}$ and $\mathcal{W}^{\prime}$ coincide except on faces of the lower and upper unit cubes, $\mathcal{W}$ contains the lower unit cube, and $\mathcal{W}^{\prime}$ the upper one.

Remark 3. The pattern $\mathcal{W}$ is obtained by taking the image of the lower unit cube under the action of $E_{1}^{*}(\sigma)$. Note that $\mathbf{w}$ and $\mathcal{W}$ do not have the same number of elements. Indeed the number of elements of the coding word $\mathbf{w}$ is equal to the sum of entries of the column with index $\mathbf{w}_{N}$ of $M_{1 \ldots N}$, whereas the number of elements of the pattern $\mathcal{W}_{i}$ is equal to the sum of entries of the line with index $i$ of $M_{1 . . N}$, by (4). Nevertheless, the number of faces in $\mathcal{W}$ is equal to $\sum_{i=1}^{3}\left|\sigma_{1 . . N}(i)\right|$.

Example 5. Let $\boldsymbol{u}=(2,2,3)$. By using Poincaré algorithm, both $\mathcal{W}$ and $\mathcal{W}^{\prime}$ have 24 faces:
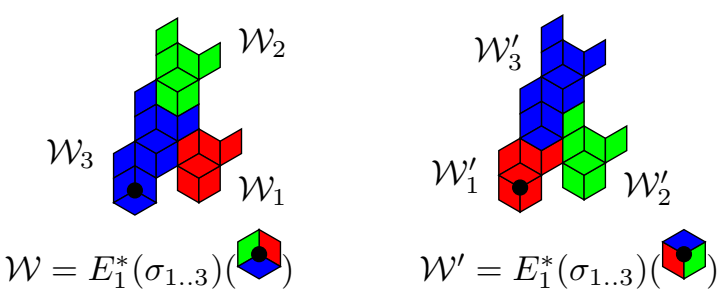

The following theorem summarizes the main properties of the dual pattern. This theorem is an adaptation to the present context of results of [2].

Theorem 1. The following properties hold:

1. $\mathcal{W} \subset \mathcal{P}_{\boldsymbol{v}}$;

2. the pattern $\mathcal{W}$ is a periodic pattern for $\mathcal{P}_{\boldsymbol{v}}$ with period vectors being

$$
\left(M_{1 . . N}\right)^{-1}\left(e_{1}-e_{2}\right),\left(M_{1 . . N}\right)^{-1}\left(e_{1}-e_{3}\right) ;
$$


3. for all $i \in\{1,2,3\}$, one has

$$
\mathcal{W}+\left(M_{1 . . N}\right)^{-1} \boldsymbol{e}_{i} \subset \mathcal{W}^{\prime}
$$

Proof. 1. We first note that the faces $\left(0, i^{*}\right) \subset \mathcal{P}_{\mathbf{1}}$ by (3) for $i=1,2,3$. We deduce the first assertion from $\boldsymbol{v}={ }^{t} M_{1 . . N} \mathbf{1}$ and from (5).

2. One has for $\left.i \neq j\left\langle\left(M_{1 . . N}\right)^{-1}\right)\left(\boldsymbol{e}_{i}-\boldsymbol{e}_{j}\right), \boldsymbol{v}\right\rangle=\left\langle\boldsymbol{e}_{i}-\boldsymbol{e}_{j}, \mathbf{1}\right\rangle=0$. Hence for every $\left.m, n \in \mathbb{Z}, \mathcal{W}+m\left(M_{1 . . N}\right)^{-1}\left(\boldsymbol{e}_{1}-\boldsymbol{e}_{2}\right)+n\left(M_{1 . . N}\right)^{-1}\right)\left(\boldsymbol{e}_{1}-\boldsymbol{e}_{3}\right) \subset \mathcal{P}_{\boldsymbol{v}}$

3. Let $\left(\boldsymbol{x}, k^{*}\right) \subset \mathcal{W}_{i}$. By definition, the face $\left(\boldsymbol{x}, k^{*}\right)$ occurs in the image by $E_{1}^{*}\left(\sigma_{1 . . N}\right)$ of the face $\left(0, i^{*}\right)$. Hence, there exists $s$ such that $\sigma_{1 . . N}(k)=p i s$. One has $\boldsymbol{x}=\left(M_{1 . . N}\right)^{-1} \boldsymbol{l}(s)$.

We assume that $p$ is not equal to the empty word. Let $j$ stand for its last letter. The face $\left(\boldsymbol{x}+\left(M_{1 . . N}\right)^{-1} \boldsymbol{e}_{i}, k^{*}\right)$ occurs in the image of the face $\left(0, j^{*}\right)$ by $E_{1}^{*}\left(\sigma_{1 . . N}\right)$, by considering as suffix $i s$. Hence it occurs in $\mathcal{W}$ and thus also in $\mathcal{W}^{\prime}$ since both sets coincide except on the lower and upper unit cubes. Assume now that $p$ is equal to the empty word. One has

$$
\begin{aligned}
\boldsymbol{x} & =\left(M_{1 . . N}\right)^{-1}\left(\boldsymbol{l}(\sigma(k))-\mathbf{e}_{i}\right)=\left(M_{1 . . N}\right)^{-1}\left(M_{1 . . N} \boldsymbol{e}_{k}-\mathbf{e}_{i}\right) \\
& =\boldsymbol{e}_{k}-\left(M_{1 . . N}\right)^{-1} \boldsymbol{e}_{i} .
\end{aligned}
$$

Hence the face $\left(\boldsymbol{x}+\left(M_{1 . . N}\right)^{-1} \boldsymbol{e}_{i}, k^{*}\right)=\left(\boldsymbol{e}_{k}, k^{*}\right)$ occurs in $\mathcal{W}^{\prime}$.

\subsection{Exchange of Pieces}

According to [2, Theorem [1 allows one to define a mapping from $\mathcal{W}$ onto $\mathcal{W}^{\prime}$ defined as an exchange of pieces between both sets.

Definition 5. We define the mapping

$$
\mathcal{E}: \mathcal{W} \rightarrow \mathcal{W}^{\prime},\left(\boldsymbol{x}, k^{*}\right) \mapsto\left(\boldsymbol{x}+\left(M_{1 . . N}\right)^{-1} \boldsymbol{e}_{i}, k^{*}\right) \text { if }\left(\boldsymbol{x}, k^{*}\right) \in \mathcal{W}_{i}
$$

This definition is illustrated in Example 5. We have seen in the proof of the third assertion of Theorem 1 that $\mathcal{E}^{k}\left(0, i^{*}\right) \in \mathcal{W}$ for $0 \leq k<\left|\sigma_{1 . . N}(i)\right|$ and that $\mathcal{E}^{k}\left(0, i^{*}\right)=\left(\boldsymbol{e}_{i}, i^{*}\right)$ for $k=\left|\sigma_{1 . . N}(i)\right|$. We define the coding of the orbit of $\left(0, i^{*}\right)$ under the action of $\mathcal{E}$ as the word of length $\left|\sigma_{1 . . N}(i)\right|$ defined over the alphabet $\{1,2,3\}^{*}$ as follows: for $1 \leq k \leq\left|\sigma_{1 . . N}(i)\right|$, its $k$ th letter is equal to the index $j$ of the subpattern $\mathcal{W}_{j}$ to which $\mathcal{E}^{k-1}\left(0, i^{*}\right)$ belongs. This word is well defined according to Assertion 3 of Theorem 1 .

Theorem 2. The coding word $\mathbf{w}=\sigma_{1 . . N}\left(\mathbf{w}_{N}\right)$ is the reversal of the coding of the orbit of the face $\left(0, \mathbf{w}_{N}^{*}\right)$ under the action of $\mathcal{E}$. The vertices of the discrete segment with coding word $\mathbf{w}$ are in a one-to-one correspondence with the faces of type $\mathbf{w}_{N}$ of $\mathcal{W}$.

Proof. We write $\mathbf{w}=z_{1} \cdots z_{k} \cdots z_{|\mathbf{w}|}$. We consider the orbit of $\left(0, \mathbf{w}_{N}^{*}\right)$ under the action of the exchange of pieces $\mathcal{E}$. The proof is done by induction on $k$. The property holds for $k=1:\left(\mathbf{0}, \mathbf{w}_{N}^{*}\right)$ belongs to $E_{1}^{*}\left(\sigma_{1 . . N}\right)\left(\mathbf{0}, z_{|\mathbf{w}|}^{*}\right)$. We assume that the induction hypothesis holds for all $\ell \leq k$ with $1 \leq k<|\mathbf{w}|$. 
Hence $\mathcal{E}^{k-1}\left(\mathbf{0}, i^{*}\right)=\left(\left(M_{1 . . N}\right)^{-1} \boldsymbol{l}\left(z_{|\mathbf{w}|-k+1} \cdots z_{|\mathbf{w}|}\right), \mathbf{w}_{N}^{*}\right)$ and $\mathcal{E}^{k-1}\left(\mathbf{0}, \mathbf{w}_{N}^{*}\right)$ is contained in $\mathcal{W}_{z_{|\mathbf{w}|-k}}$. Consequently, $\mathcal{E}^{k}\left(0, \mathbf{w}_{N}^{*}\right)=\mathcal{E}^{k-1}\left(0, \mathbf{w}_{N}^{*}\right)+M_{1 . . N}^{-1} \boldsymbol{e}_{z_{|\mathbf{w}|-k}}$ and $\mathcal{E}^{k-1}\left(\mathbf{0}, i^{*}\right)=\left(M_{1 . . N}^{-1} \boldsymbol{l}\left(z_{1} \cdots z_{|\mathbf{w}|-k}\right), \mathbf{w}_{N}^{*}\right)$. The one-to-one mapping comes from $\left\langle-M_{1 . . N}^{-1} \boldsymbol{l}\left(z_{1} \cdots z_{k}\right)+\boldsymbol{u}_{N}, \boldsymbol{v}\right\rangle=\left\langle-\boldsymbol{l}\left(z_{1} \cdots z_{k}\right)+\boldsymbol{l}(\mathbf{w}), \mathbf{1}\right\rangle$.

Example 6 . Let $\boldsymbol{u}=(2,2,3)$ on which Poincaré algorithm is applied, and let

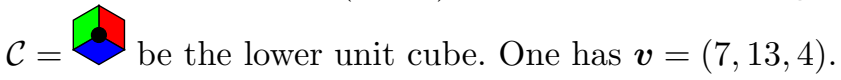

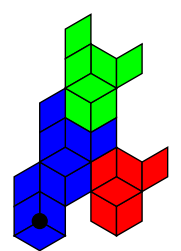

$\mathcal{W}=E_{1}^{*}\left(\sigma_{1 . .3}\right)(\mathcal{C})$

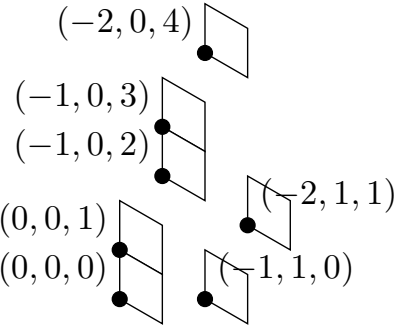

Vertices of the faces of type 1

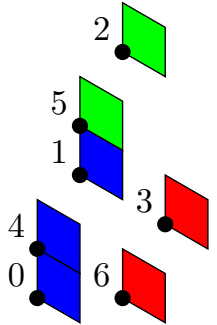

Scalar product with $v$

The letters of $\mathbf{w}=1231233$ correspond to the color of the faces of type 1 of $\mathcal{W}$ ordered decreasingly by their scalar product with $\boldsymbol{v}$. The vertices of the discrete segment depicted in Example 3 .

$$
(0,0,0),(1,0,0),(1,1,0),(1,1,1),(2,1,1),(2,2,1),(2,2,2),(2,2,3)
$$

are in one-to-one correspondence with the vertices of the faces of type 1 by the map

$$
\boldsymbol{x} \mapsto-\left(M_{1 . .3}\right)^{-1} \cdot \boldsymbol{x}+\boldsymbol{u}_{N}=\left(\begin{array}{rrr}
-2 & 0 & 1 \\
1 & -1 & 0 \\
0 & 3 & -2
\end{array}\right) \cdot \boldsymbol{x}+\left(\begin{array}{l}
1 \\
0 \\
0
\end{array}\right) \text {. }
$$

Remark 4. Theorem 2 does not only apply for $\mathbf{w}_{N}$ but also for the other letters. Note that it allows a labelling of faces of a given type by increasing distance to the Euclidean plane with normal vector $\boldsymbol{v}$. Theorem 2 can be considered as an analogue of the description of Sturmian and Christoffel words in terms of codings of rotations acting on the unit circle. It also provides a second simple generation method for discrete segments.

\section{Conclusion}

We have described here a generation method for discrete segments connecting the origin to a given point $\left(u_{1}, u_{2}, u_{3}\right) \in \mathbb{N}^{3}$. We obtain two generation methods: the first one is stated in terms of an iteration of a finite number of substitutions governed by the choice of the underlying three-dimensional Euclid's algorithm (see Section 3); the second one is of a more geometric flavor and involves a dual discrete plane (see Section 4.4). We recover here duality ideas that can be found in [7] in the framework of Christoffel words. Our contribution mostly relies in the 
application and development of the formalism of [2] in the context of the study of discrete lines. Note that the use of generalized substitutions (see Section 2.2) associated with multi-dimensional continued fraction algorithms has also already proved its efficiency in discrete geometry for the generation of discrete planes, see [116]. We now aim at starting a thorough investigation and comparison of the generation properties of the most classical three-dimensional Euclid's algorithm.

\section{References}

1. Andres, E.: Discrete linear objects in dimension $n$ : the standard model. Graphical Models 65, 92-111 (2003)

2. Arnoux, P., Ito, S.: Pisot substitutions and Rauzy fractals. Bull. Belg. Math. Soc. Simon Stevin 8(2), 181-207 (2001)

3. Arnoux, P., Mauduit, C., Shiokawa, I., Tamura, J.I.: Complexity of sequences defined by billiards in the cube. Bull. Soc. Math. France 122, 1-12 (1994)

4. Arnoux, P., Rauzy, G.: Représentation géométrique de suites de complexité $2 n+1$. Bull. Soc. Math. France 119, 199-215 (1991)

5. Berthé, V., Ferenczi, S., Zamboni, L.Q.: Interactions between dynamics, arithmetics and combinatorics: the good, the bad, and the ugly. In: Algebraic and Topological Dynamics, Contemp. Math., vol. 385, pp. 333-364. Amer. Math. Soc., Providence (2005)

6. Berthé, V., Lacasse, A., Paquin, G., Provençal, X.: A study of Jacobi-Perron boundary words for the generation of discrete planes. Preprint (2010)

7. Berthé, V., de Luca, A., Reutenauer, C.: On an involution of Christoffel words and Sturmian morphisms. European Journal of Combinatorics 29, 535-553 (2008)

8. Brimkov, V.E., Barneva, R.P., Brimkov, B., de Vieilleville, F.: Offset approach to defining 3D digital lines. In: Advances in Visual Computing, pp. 678-687 (2008)

9. Brimkov, V.E., Barneva, R.P., Brimkov, B.: Minimal offsets that guarantee maximal or minimal connectivity of digital curves in $n \mathrm{D}$. In: Brlek, S., Reutenauer, C., Provençal, X. (eds.) DGCI 2009. LNCS, vol. 5810, pp. 337-349. Springer, Heidelberg (2009)

10. Broise-Alamichel, A., Guivarc'h, Y.: Exposants caractéristiques de l'algorithme de Jacobi-Perron et de la transformation associée. Ann. Inst. Fourier (Grenoble) 51(3), 565-686 (2001)

11. Fernique, T.: Generation and recognition of digital planes using multi-dimensional continued fractions. In: Coeurjolly, D., Sivignon, I., Tougne, L., Dupont, F. (eds.) DGCI 2008. LNCS, vol. 4992, pp. 33-44. Springer, Heidelberg (2008)

12. Figueiredo, O., Reveillès, J.P.: New results about 3d digital lines. In: Proc. Internat. Conference Vision Geometry V, Proc. SPIE, vol. 2826, pp. 98-108 (1996)

13. Hubert, P.: Suites équilibrées. Theoret. Comput. Sci. 242, 91-108 (2000)

14. Klette, R., Rosenfeld, A.: Digital straightness-a review. Discrete Applied Mathematics 139, 197-230 (2004)

15. Lothaire, M.: Algebraic Combinatorics on Words. Cambridge University Press, Cambridge (2002)

16. Reveillès, J.P.: Géométrie discrète, calcul en nombres entiers et algorithmique. Thèse de Doctorat, Université Louis Pasteur, Strasbourg (1991)

17. Schweiger, F.: Multidimensinal Continued Fraction. Oxford Univ. Press, New York (2000)

18. Toutant, J.L.: Characterization of the closest discrete approximation of a line in the 3-Dimensional space. In: Advances in Visual Computing, pp. 618-627 (2006) 